EPJ Web of Conferences 81, 05009 (2014)

DOI: $10.1051 /$ epjconf/20148105009

(C) Owned by the authors, published by EDP Sciences, 2014

\title{
Decays of open charmed mesons in the extended Linear Sigma Model
}

\author{
Walaa I. Eshraim ${ }^{1,}$ a and Francesco Giacosa ${ }^{1,2}$ \\ ${ }^{1}$ Institute for Theoretical Physics, Goethe University, Max-von-Laue-Str. 1, D-60438 Frankfurt am Main, \\ Germany \\ ${ }^{2}$ Institute of Physics, Jan Kochanowski University, 25-406 Kielce, Poland
}

\begin{abstract}
We enlarge the so-called extended linear Sigma model (eLSM) by including the charm quark according to the global $U(4)_{r} \times U(4)_{l}$ chiral symmetry. In the eLSM, besides scalar and pseudoscalar mesons, also vector and axial-vector mesons are present. Almost all the parameters of the model were fixed in a previous study of mesons below $2 \mathrm{GeV}$. In the extension to the four-flavor case, only three additional parameters (all of them related to the bare mass of the charm quark) appear. We compute the (OZI dominant) strong decays of open charmed mesons. The results are compatible with the experimental data, although the theoretical uncertainties are still large.
\end{abstract}

\section{Introduction}

Charm physics is an experimentally and theoretically active field of hadronic physics [1]. The study of strong decays of the heavy mesons into light pseudoscalar mesons is useful to classify the charmed states. In this paper we study the OZI-dominant decays of open charmed mesons by using the extended linear sigma model (eLSM).

The eLSM is a chiral model which describes successfully the vacuum phenomenology of light mesons such as masses and decay widths [2,3]. The eLSM Lagrangian is invariant under chiral symmetry $U\left(N_{f}\right)_{R} \times U\left(N_{f}\right)_{L}\left(N_{f}=2\right.$ in Ref.[2] and $N_{f}=3$ in Ref.[3]) and dilatation transformations. Chiral symmetry is broken explicitly by non-vanishing quark masses and spontaneously by a non-zero quark condensate in the QCD vacuum.

In this paper we extend the eLSM by including the charm degree of freedom, thus working with four flavor $\left(N_{f}=4\right)[4,5]$. The masses of open and hidden charmed mesons have been calculated $[4,5]$ and turn out to be compatible with the experimental data [6]. Here we then concentrate on the decay widths of open charmed mesons [5].

\section{The eLSM and its implications}

In order to extend the eLSM to the case of four flavour, $N_{f}=4$, we introduce the (pseudo)scalar and (axial-)vector meson fields in terms of $4 \times 4$ (instead of $3 \times 3)$ matrices, which the charmed mesons

a. Based on the poster preperation given by W. I.Eshraim at the 13th International Workshop on Meson Production, Properties and Interaction, 29th May - 3rd June 2014, KRAKÓW, POLAND. The poster was awarded with the special prize of the Poster Session. e-mail: weshraim@th.physik.uni-frankfurt.de 
appear in the fourth row and column. The multiplet of (pseudo)scalar states is given by

$$
\Phi=S+i P=\frac{1}{\sqrt{2}}\left(\begin{array}{cccc}
\frac{\left(\sigma_{N}+a_{0}^{0}\right)+i\left(\eta_{N}+\pi^{0}\right)}{\sqrt{2}} & a_{0}^{+}+i \pi^{+} & K_{0}^{*+}+i K^{+} & D_{0}^{* 0}+i D^{0} \\
a_{0}^{-}+i \pi^{-} & \frac{\left(\sigma_{N}-a_{0}^{0}\right)+i\left(\eta_{N}-\pi^{0}\right)}{\sqrt{2}} & K_{0}^{* 0}+i K^{0} & D_{0}^{*-}+i D^{-} \\
K_{0}^{*-}+i K^{-} & \bar{K}_{0}^{* 0}+i \bar{K}^{0} & \sigma_{S}+i \eta_{S} & D_{S 0}^{*-}+i D_{S}^{-} \\
\bar{D}_{0}^{* 0}+i \bar{D}^{0} & D_{0}^{*+}+i D^{+} & D_{S 0}^{*+}+i D_{S}^{+} & \chi_{C 0}+i \eta_{C}
\end{array}\right),
$$

and the left-handed and right-handed matrices containing the vector fields and axial-vector fields are given by

$$
L^{\mu}\left(R^{\mu}\right)=V^{\mu} \pm A^{\mu}=\frac{1}{\sqrt{2}}\left(\begin{array}{cccc}
\frac{\omega_{N}+\rho^{0}}{\sqrt{2}} \pm \frac{f_{1 N}+a_{1}^{0}}{\sqrt{2}} & \rho^{+} \pm a_{1}^{+} & K^{*+} \pm K_{1}^{+} & D^{* 0} \pm D_{1}^{0} \\
\rho^{-} \pm a_{1}^{-} & \frac{\omega_{N}-\rho^{0}}{\sqrt{2}} \pm \frac{f_{1 N}-a_{1}^{0}}{\sqrt{2}} & K^{* 0} \pm K_{1}^{0} & D^{*-} \pm D_{1}^{-} \\
K^{*-} \pm K_{1}^{-} & \bar{K} \bar{K}_{1}^{0} & \omega_{S} \pm f_{1 S} & D_{S}^{*-} \pm D_{S 1}^{-} \\
\bar{D}^{* 0} \pm \bar{D}_{1}^{0} & D^{*+} \pm D_{1}^{+} & D_{S}^{*+} \pm D_{S 1}^{+} & J / \psi \pm \chi_{C 1}
\end{array}\right)^{\mu}
$$

The charmed fields $D^{* 0}, D^{*}, D_{0}^{* 0}, D_{0}^{*}, D_{S 0}^{*}, D_{1}, D_{S 1}, \chi_{c 1}, \chi_{c 0}$, and $J / \psi$ are assigned to the $q \bar{q}$ resonances $D^{*}(2007)^{0}, D^{*}(2010)^{ \pm}, D_{0}^{*}(2400)^{0}, D_{0}^{*}(2400)^{ \pm}, D_{S 0}^{*}(2317), D_{1}(2420), D_{S 1}(2536)$ $\chi_{c 1}(1 P), \chi_{c 0}(1 P)$, and $J / \psi(1 S)$, respectively, see the details in Ref. [5]. The explicit form of the eLSM Lagrangian for $N_{f}=4$ is analogous to the case $N_{f}=3$ of Ref. [3] (but has an additional term $\left.-2 \operatorname{Tr}\left[E \Phi^{\dagger} \Phi\right]\right)$ :

$$
\begin{aligned}
\mathcal{L} & =\frac{1}{2}\left(\partial_{\mu} G\right)^{2}-\frac{1}{4} \frac{m_{G}^{2}}{\Lambda^{2}}\left(G^{4} \log \frac{G}{\Lambda}-\frac{G^{4}}{4}\right)+\operatorname{Tr}\left[\left(D^{\mu} \Phi\right)^{\dagger}\left(D^{\mu} \Phi\right)\right]-m_{0}^{2}\left(\frac{G}{G_{0}}\right)^{2} \operatorname{Tr}\left(\Phi^{\dagger} \Phi\right)-\lambda_{1}\left[\operatorname{Tr}\left(\Phi^{\dagger} \Phi\right)\right]^{2} \\
& -\lambda_{2} \operatorname{Tr}\left(\Phi^{\dagger} \Phi\right)^{2}+\operatorname{Tr}\left[H\left(\Phi+\Phi^{\dagger}\right)\right]+\operatorname{Tr}\left\{\left[\left(\frac{G}{G_{0}}\right)^{2} \frac{m_{1}^{2}}{2}+\Delta\right]\left[\left(L^{\mu}\right)^{2}+\left(R^{\mu}\right)^{2}\right]\right\}-\frac{1}{4} \operatorname{Tr}\left[\left(L^{\mu \nu}\right)^{2}+\left(R^{\mu \nu}\right)^{2}\right] \\
& -2 \operatorname{Tr}\left[E \Phi^{\dagger} \Phi\right]+c\left(\operatorname{det} \Phi-\operatorname{det} \Phi^{\dagger}\right)^{2}+i \frac{g_{2}}{2}\left\{\operatorname{Tr}\left(L_{\mu \nu}\left[L^{\mu}, L^{\nu}\right]\right)+\operatorname{Tr}\left(R_{\mu v}\left[R^{\mu}, R^{\nu}\right]\right)\right\} \\
& +\frac{h_{1}}{2} \operatorname{Tr}\left(\Phi^{\dagger} \Phi\right) \operatorname{Tr}\left[\left(L^{\mu}\right)^{2}+\left(R^{\mu}\right)^{2}\right]+h_{2} \operatorname{Tr}\left[\left(\Phi R^{\mu}\right)^{2}+\left(L^{\mu} \Phi\right)^{2}\right]+2 h_{3} \operatorname{Tr}\left(\Phi R_{\mu} \Phi^{\dagger} L^{\mu}\right)+\ldots
\end{aligned}
$$

where $G$ is the dilaton field, $D^{\mu} \Phi \equiv \partial^{\mu} \Phi-i g_{1}\left(L^{\mu} \Phi-\Phi R^{\mu}\right), L^{\mu v} \equiv \partial^{\mu} L^{v}-\partial^{v} L^{\mu}$, and $R^{\mu \nu} \equiv$ $\partial^{\mu} R^{v}-\partial^{\nu} R^{\mu}$. The terms $\operatorname{Tr}\left[H\left(\Phi+\Phi^{\dagger}\right)\right]$ with $H=1 / 2 \operatorname{diag}\left\{h_{0 N}, h_{0 N}, \sqrt{2} h_{0 S}, \sqrt{2} h_{0 C}\right\},-2 \operatorname{Tr}\left[E \Phi^{\dagger} \Phi\right]$ with $E=\operatorname{diag}\left\{\varepsilon_{N}, \varepsilon_{N}, \varepsilon_{S}, \varepsilon_{C}\right\}, \varepsilon_{i} \propto m_{i}^{2}, \varepsilon_{N}=\varepsilon_{S}=0$, and $\operatorname{Tr}\left[\Delta\left(L^{\mu 2}+R^{\mu 2}\right)\right]$ with $\delta=$ $\operatorname{diag}\left\{\delta_{N}, \delta_{N}, \delta_{S}, \delta_{C}\right\}, \delta_{i} \sim m_{i}^{2}, \delta_{N}=\delta_{S}=0$, break chiral symmetry due to nonzero quark masses and are especially important for mesons containing the charm quark. When $m_{0}^{2}<0$ spontaneous symmetry breaking occurs and the fields $\sigma_{N}, \sigma_{S}$, and $\chi_{C 0}$ condense [3-5]. Most of the parameters of the model were already fixed in the three-flavor study of Ref. [3]. Only three new parameters appear and all of them are related to the bare mass of the charm quark. They were determined in Ref. [5] through a fit to the masses of charmed mesons. As an outcome, the charm-anticharm condensate is sizable, $\left\langle\chi_{C 0}\right\rangle=178 \pm 28 \mathrm{MeV}$.

\section{Results and Conclusion}

We present the results of the decay widths of open charmed mesons in Table 1. Although the theoretical errors are in some channels very large, the results are compatible with the experiment [6]. 
MESON 2014-13 $3^{\text {th }}$ International Workshop on Production, Properties and Interaction of Mesons

Table 1. Decay widths of open charmed mesons

\begin{tabular}{lll}
\hline Decay Channel & Theoretical results [MeV] & Experimental results [MeV] (from [6] $)$ \\
\hline$D_{0}^{*}(2400)^{0} \rightarrow D \pi$ & $139_{-114}^{+243}$ & $D^{+} \pi^{-}$seen; full width $\Gamma=267 \pm 40$ \\
$D_{0}^{*}(2400)^{+} \rightarrow D \pi$ & $51_{-51}^{+182} \rightarrow 0.003$ & $D^{+} \pi^{0}$ seen; full width: $\Gamma=283 \pm 24 \pm 34$ \\
$D^{*}(2007)^{0} \rightarrow D^{0} \pi^{0}$ & $0.025 \pm 0.34$ & seen; $<1.3$ \\
$D^{*}(2007)^{0} \rightarrow D^{+} \pi^{-}$ & 0 & not seen \\
$D^{*}(2010)^{+} \rightarrow D^{+} \pi^{0}$ & $0.018_{-0.003}^{+0.002}$ & $0.029 \pm 0.008$ \\
$D^{*}(2010)^{+} \rightarrow D^{0} \pi^{+}$ & $0.038_{-0.004}^{+0.005}$ & $0.065 \pm 0.017$ \\
$D_{1}(2420)^{0} \rightarrow D^{*} \pi$ & $65_{-37}^{+51}$ & $D^{*+} \pi^{-}$seen; full width: $\Gamma=27.4 \pm 2.5$ \\
$D_{1}(2420)^{0} \rightarrow D^{0} \pi \pi$ & $0.59 \pm 0.02$ & seen \\
$D_{1}(2420)^{0} \rightarrow D^{+} \pi^{-} \pi^{0}$ & $0.21_{-0.015}^{+0.01}$ & - \\
$D_{1}(2420)^{0} \rightarrow D^{+} \pi^{-}$ & 0 & not seen; $\Gamma\left(D^{+} \pi^{-}\right) / \Gamma\left(D^{*+} \pi^{-}\right)<0.24$ \\
$D_{1}(2420)^{+} \rightarrow D^{*} \pi$ & $65_{-36}^{+51}$ & $D^{* 0} \pi^{+}$seen; full width: $\Gamma=25 \pm 6$ \\
$D_{1}(2420)^{+} \rightarrow D^{+} \pi \pi$ & $0.56 \pm 0.02$ & seen \\
$D_{1}(2420)^{+} \rightarrow D^{0} \pi^{0} \pi^{+}$ & $0.22 \pm 0.01$ & - \\
$D_{1}(2420)^{+} \rightarrow D^{0} \pi^{+}$ & 0 & not seen; $\Gamma\left(D^{0} \pi^{+}\right) / \Gamma\left(D^{* 0} \pi^{+}\right)<0.18$ \\
\hline
\end{tabular}

Moreover, the decay of the vector and axial-vector chiral partners $D^{*}(2010)$ and $D_{1}(2420)$ are well described. This fact shows that chiral symmetry is still important for charmed mesons. Studies in progress are the calculation of the decay widths of the charmonia and the mixing of axial-vector and pseudovector charmed states.

\section{Acknowledgements}

The authors thank D. Parganlija and D. H. Rischke for useful discussions. W.I.E. acknowledges financial support from DAAD.

\section{References}

[1] N. Brambilla, A. Pineda, J. Soto and A. Vairo, Rev. Mod. Phys. 77 (2005) 1423.

[2] D. Parganlija, F. Giacosa and D. H. Rischke, Phys. Rev. D 82, 054024 (2010).

[3] D. Parganlija, P. Kovacs, G. Wolf, F. Giacosa and D. H. Rischke, Phys. Rev. D 87, 014011 (2013).

[4] W. I. Eshraim, PoS QCD -TNT-III, 049 (2013) [arXiv:1401.3260 [hep-ph]].

[5] W. I. Eshraim, F. Giacosa and D. H. Rischke, arXiv:1405.5861 [hep-ph].

[6] J. Beringer et al. (particle Data Group), Phys. Rev. D 86, 010001 (2012). 